\title{
Triamcinolone Acetonide Extended-Release: A Review in Osteoarthritis Pain of the Knee
}

\author{
Julia Paik $^{1}$ - Sean T. Duggan ${ }^{1}$. Susan J. Keam ${ }^{1}$
}

Published online: 8 March 2019

๑) Springer Nature 2019, corrected publication 2019

\begin{abstract}
Triamcinolone acetonide extended-release (ER) $32 \mathrm{mg}$ (Zilretta ${ }^{\circledR}$ ) is approved in the USA for the management of osteoarthritis (OA) pain of the knee and is administered as a single, $5 \mathrm{~mL}$ intra-articular (IA) injection. Although the therapeutic effects from IA corticosteroids are typically short-lived, triamcinolone acetonide ER is formulated in poly (lactic-co-glycolic acid) (PLGA) microspheres that slowly release triamcinolone acetonide in the synovium, enabling their prolonged presence in the joint. This reduces systemic exposure and lessens corticosteroid-related systemic adverse reactions, such as blood glucose elevations. In a 24-week, randomized, phase III clinical trial, triamcinolone acetonide ER $32 \mathrm{mg}$ significantly improved mean average daily pain intensity in patients with knee OA relative to placebo, and pain, stiffness and physical function (according to WOMAC criteria) relative to placebo and triamcinolone acetonide crystalline suspension (CS). Triamcinolone acetonide ER was generally well tolerated, with a tolerability profile similar to that of triamcinolone acetonide CS and placebo. Findings from a single-arm phase IIIb study indicated that a repeat administration of triamcinolone acetonide ER may be similarly efficacious to an initial injection without having deleterious effects on cartilage or other aspects of joint structure. Thus, triamcinolone acetonide ER expands the treatment options available for the management of OA pain of the knee.
\end{abstract}

Triamcinolone acetonide ER: clinical

considerations in OA pain of the knee

Allows prolonged synovial presence and lower systemic absorption of triamcinolone acetonide

Associated with significantly lower blood glucose elevations than triamcinolone acetonide CS

Significantly improves pain in patients with knee OA

Generally well tolerated

The manuscript was reviewed by: $\boldsymbol{H}$. S. B. Baraf, Center for Rheumatology and Bone Research, Arthritis and Rheumatism Associates, Wheaton, MD, USA; $\boldsymbol{S}$. R. Kingsbury, Leeds Institute of Rheumatic and Musculoskeletal Medicine and NIHR Leeds Biomedical Research Centre, University of Leeds, Leeds, UK.

Julia Paik

demail@springer.com

1 Springer, Private Bag 65901, Mairangi Bay, Auckland 0754, New Zealand

\section{Introduction}

Accounting for most of the total global burden of osteoarthritis (OA) [1], knee OA has become increasingly prevalent with growing obesity rates and the aging population [2]. Pain from knee OA is highly debilitating, with underlying consequences being the impact of pain on mobility, mood and sleep [3]. A positive correlation has also been found between the severity of walking disability from OA and a risk of death (mostly attributed to cardiovascular disease) $[2,4]$.

Current evidence suggests that synovial inflammation plays a significant role in the pathophysiology of knee OA, making the synovium a key target for treatment [5]. Accordingly, intra-articular (IA) injections of corticosteroids, such as triamcinolone acetonide, have been used for a number of decades to treat OA pain, with their likely mode of action being modulation of synovial inflammation, given their potent anti-inflammatory properties [6-8]. However, the therapeutic efficacy from IA corticosteroids is relatively short-lived, with their clinically meaningful benefits only lasting up to 4 weeks postadministration [9]. These shortlived benefits are thought to be due to a rapid drug efflux of the corticosteroid from the joint into the systemic circulation, leading to corticosteroid-related adverse reactions, 
and is also thought to promote a transient elevation in blood glucose levels (lasting a few days postinjection) in patients with type 2 diabetes mellitus (T2DM), an effect that is seen shortly after IA corticosteroid administration [10].

To overcome the efficacy limitations and the corticosteroid-related systemic adverse effects of conventional IA corticosteroid formulations, a novel, extended-release (ER), poly (lactic-co-glycolic acid) (PLGA) microsphere-based formulation of triamcinolone acetonide as an IA injection (Zilretta ${ }^{\circledR}$ ) has been developed and is approved in the USA for the management of OA pain of the knee [11]. This review evaluates the pharmacological features of triamcinolone acetonide ER and its therapeutic efficacy and tolerability in this indication, focusing on data relevant to the approved dosage of $32 \mathrm{mg}$.

\section{Pharmacodynamic Properties}

Triamcinolone acetonide ER is formulated in 75:25 (lactic to glycolic acid) PLGA microspheres $\approx 45$ microns in diameter, with small triamcinolone acetonide crystals embedded throughout the PLGA matrix [nominal drug load of 25\% $(w / w)][11,12]$. Following administration, biodegradation of the PLGA microspheres (via hydrolysis of ester linkages [13]) begins with the formation of small channels $(\approx 500 \mathrm{~nm}$ diameter) on their surface, which allows slow drug release [14]. The slow and steady degradation of the PLGA microspheres is facilitated by their lower glycolic acid content [15] and their small size ( $<300$ microns), which fosters homogeneous PLGA degradation [13]. The PLGA matrices ultimately degrade into oligomeric poly-acid units before forming lactic and glycolic acids, which are then eliminated as carbon dioxide and water [14]. A recent in vitro study [16] has shown that adding local anaesthetics (lidocaine, ropivacaine and/or bupivacaine) when preparing $5 \mathrm{~mL}$ of triamcinolone acetonide ER does not negatively affect the physicochemical properties of triamcinolone acetonide ER, including $\mathrm{pH}$, syringeability, particle size, agglomeration and purity.

As a synthetic glucocorticoid, triamcinolone acetonide binds to and activates the glucocorticoid receptor to produce anti-inflammatory responses, such as down-regulating proinflammatory cytokine expression [17], preventing prostaglandin and leukotriene synthesis and release of arachidonic acid, and activating anti-inflammatory transcription factors such as lipocortins [11].

Although corticosteroid-induced suppression of the hypothalamic-pituitary-adrenal axis is reversible, adrenal insufficiency may occur after corticosteroid withdrawal [11]. Following a single IA injection of triamcinolone acetonide ER $32 \mathrm{mg}$, adrenal suppression occurred within 12-24 h and gradually returned to baseline levels within 30-42 days [11]. The extent of adrenal suppression from triamcinolone acetonide ER was generally consistent to that of the commonly used triamcinolone acetonide crystalline suspension (CS) formulation [18]. Both triamcinolone acetonide ER $40 \mathrm{mg}$ and triamcinolone acetonide CS $40 \mathrm{mg}$ were associated with significant reductions in $24 \mathrm{~h}$ weighted mean serum cortisol (maximum reduction within $24 \mathrm{~h}$ of administration of 42.7 vs. $59.0 \%$ ) and $24 \mathrm{~h}$ urinary cortisol excretion (43.8 vs. $58.5 \%$ ) from baseline. There were no significant differences between the two formulations in these parameters at $24 \mathrm{~h}$, 2 weeks and 6 weeks posttreatment [18].

Previous clinical studies have suggested that IA corticosteroids may be chondrotoxic; however, this is only likely with greater exposure to the drug and the risk of toxicity is generally outweighed by the clinical benefits [19]. In a rat model of repeated localized knee arthritis, triamcinolone acetonide ER appeared to prevent cartilage damage [20]. Although triamcinolone acetonide ER decreased glycosaminoglycan synthesis in animal studies, this was fully reversible, with normal synthesis evident 9 months after administration [21,22] and cellular signs of repair where there was articular cartilage damage [22].

In patients with T2DM (managed with stable doses of oral hypoglycaemic agents) and symptomatic knee OA, triamcinolone acetonide ER had less disruption of glycaemic control than triamcinolone acetonide CS following IA administration in a phase II study [23]. Patients treated with IA triamcinolone acetonide ER $32 \mathrm{mg}(n=18)$ had significantly $(p=0.045)$ smaller blood glucose elevations than those treated with IA triamcinolone acetonide CS $40 \mathrm{mg}(n=15)$ [change in average daily continuous glucose monitoring glucose (CGMG) at $72 \mathrm{~h}$ from baseline of $14.7 \mathrm{vs} .33 .9 \mathrm{mg} / \mathrm{dL}$, least squares mean (LSM) difference $-19.2 \mathrm{mg} / \mathrm{dL}$ ] [23]. The mean average daily CGMG increases over $72 \mathrm{~h}$ from baseline with triamcinolone acetonide ER and triamcinolone acetonide CS were 8.2 and $37.1 \mathrm{mg} / \mathrm{dL}$ [23]. In addition, the mean blood glucose level elevation experienced by triamcinolone acetonide ER recipients during the first three days following treatment (CGMG from $155.24-163.4 \mathrm{mg} / \mathrm{dL}$ ) remained within the target glycaemic range $(70-180 \mathrm{mg} / \mathrm{dL})$, unlike that of triamcinolone acetonide CS recipients (from 161.71-198.8 mg/dL). Post hoc analyses also showed that there was a significant $(p=0.035)$ within-group increase in CGMG with triamcinolone acetonide CS but not with triamcinolone acetonide ER, suggesting that triamcinolone acetonide ER may not affect glycaemic control in patients with type 2 diabetes mellitus [23]. These differences may be attributed a lower systemic exposure to triamcinolone acetonide following treatment with triamcinolone acetonide ER compared with triamcinolone acetonide CS (Sect. 3).

\section{Pharmacokinetic Properties}

Triamcinolone acetonide had a markedly longer presence in the knee synovial fluid when administered as triamcinolone 
acetonide ER $32 \mathrm{mg}$ than triamcinolone acetonide CS $40 \mathrm{mg}$ in a phase II pharmacokinetic study [24]. Synovial triamcinolone acetonide concentrations were sustained above the lower limits of quantification (LLOQ) $(0.05 \mathrm{ng} / \mathrm{mL})$ up to week 12 after IA administration of triamcinolone acetonide ER $32 \mathrm{mg}$, whereas concentrations were below the LLOQ at week 6 after IA triamcinolone acetonide CS $40 \mathrm{mg}$ administration. Following a $5 \mathrm{~mL}$ IA injection of triamcinolone acetonide ER $32 \mathrm{mg}$, the mean synovial triamcinolone acetonide concentration reached $231.1 \mathrm{ng} / \mathrm{mL}$ by the end of week 1 before declining to $3.6 \mathrm{ng} / \mathrm{mL}$ at week 6 and $0.3 \mathrm{ng} /$ $\mathrm{mL}$ at week 12 . By contrast, the mean concentration of triamcinolone acetonide ER 6 weeks after treatment with $1 \mathrm{~mL}$ triamcinolone acetonide CS $40 \mathrm{mg}$ was $0.0077 \mathrm{ng} / \mathrm{mL}$ [24].

Triamcinolone acetonide plasma concentrations are lower and systemic exposure is longer after administration of IA triamcinolone acetonide ER than IA triamcinolone acetonide CS [11, 24]. Although the median time to peak plasma triamcinolone acetonide concentration $\left(\mathrm{C}_{\max }\right)$ with triamcinolone acetonide ER was similar with that of triamcinolone acetonide $\mathrm{CS}$, the mean $\mathrm{C}_{\max }$ plasma triamcinolone acetonide concentration with triamcinolone acetonide ER was more than 18 times lower than that with triamcinolone acetonide CS (Table 1) [11]. In the phase II pharmacokinetics study, the mean plasma concentration of triamcinolone acetonide at $24 \mathrm{~h}$ post-administration was low and virtually unchanged from peak levels with triamcinolone acetonide ER $(0.8 \mathrm{ng} / \mathrm{mL})$, while that with triamcinolone acetonide CS had dropped by more than $50 \%$ from the $\mathrm{C}_{\max }$ (to $5.0 \mathrm{ng}$ / $\mathrm{mL}$ ) [24]. Systemic exposure to triamcinolone acetonide [measured using the area under the curve (AUC)] following IA triamcinolone acetonide ER was over ten times lower at $24 \mathrm{~h}$ than that of IA triamcinolone acetonide CS, and the AUC from baseline to infinity $\left(\mathrm{AUC}_{\infty}\right)$ suggested that the total drug exposure after triamcinolone acetonide ER administration was slightly over half that seen with triamcinolone acetonide CS (Table 1). The mean plasma half-life of triamcinolone acetonide after IA triamcinolone acetonide ER was approximately four times longer than that seen with triamcinolone acetonide CS (Table 1) and the mean residence time was more than seven times longer (453.7 vs. $60.9 \mathrm{~h}$ ). Systemic clearance was very slow in both treatments
(0.0001 and $0.0000 \mathrm{~mL} / \mathrm{h} / \mathrm{kg}$ for triamcinolone acetonide ER and triamcinolone acetonide CS) [24].

Three metabolites of triamcinolone acetonide have been identified through animal studies ( $6 \beta$-hydroxytriamcinolone acetonide, 21-carboxytriamcinolone acetonide and 21 -carboxy-6 $\beta$-hydroxytriamcinolone acetonide), all of which are likely to be considerably less active than the parent compound, given their structures (e.g. increased water solubility favouring rapid elimination) [25].

\section{Therapeutic Efficacy}

\subsection{Single Administration}

The therapeutic efficacy of IA triamcinolone acetonide ER in patients with OA pain of the knee has been evaluated in three randomized, double-blind, multicentre studies, of which two were dose-ranging phase II $(n=228)$ [26] or IIb $(n=306)$ [27] trials and the other a phase III trial $(n=484)$ [28]. This section focuses on the trials $[27,28]$ that evaluated the dose $(32 \mathrm{mg})$ and injection volume $(5 \mathrm{~mL})$ of triamcinolone acetonide ER that was approved in the USA for the management of OA pain of the knee [11].

Eligible patients in these studies had symptomatic knee $\mathrm{OA}$ as per American College of Rheumatology (ACR) criteria for $\geq 6$ months prior to screening, a Kellgren-Lawrence grade of 2 or 3 as assessed on a screening radiograph, patientreported pain in the index knee for $>15$ days in the previous month, and a mean average daily pain (ADP) intensity score of 5-9, specified in the phase III study to be lasting $\geq 5$ days during the week before treatment $[27,28]$. In the phase III trial, exclusion criteria included arthroscopic or open surgery of the index knee within 12 months of screening and patients with diabetes with a haemoglobin A1c level of $>7.5 \%$ [28]. In the phase IIb trial, patients with other arthritic or immunemediated inflammatory disorders $<12$ months before screening were excluded [27]. Common exclusion criteria across the clinical trials included the recent use of IA, intramuscular, or oral corticosteroids, or other IA investigational drugs [27, 28].

The primary endpoint was the LSM change from baseline to week 12 in ADP intensity scores for triamcinolone acetonide

Table 1 Mean plasma pharmacokinetic parameters following IA administration of $5 \mathrm{~mL}$ triamcinolone acetonide ER $32 \mathrm{mg}$ or $1 \mathrm{~mL}$ triamcinolone acetonide CS $40 \mathrm{mg}(n=78)$ [11]

\begin{tabular}{lllll} 
Treatment & $\mathrm{t}_{\max }{ }^{\mathrm{a}}(\mathrm{h})$ & $\mathrm{C}_{\max }(\mathrm{ng} / \mathrm{mL})$ & $\mathrm{AUC}_{24}(\mathrm{ng} \bullet \mathrm{h} / \mathrm{mL})$ & $\mathrm{AUC} \boldsymbol{\infty}_{\infty}(\mathrm{ng} \bullet \mathrm{h} / \mathrm{mL})$ \\
\hline TA-ER & 7.00 & 1.14 & 21.22 & 845.15 \\
TA-CS & 6.00 & 21.06 & 297.55 & 1567.57 \\
\hline
\end{tabular}

$A U C$ area under the curve, $C_{\max }$ maximum concentration, $C S$ crystalline suspension, $E R$ extended-release, $I A$ intra-articular, $t_{1 / 2}$ half-life, $T A$ triamcinolone acetonide, $t_{\max }$ time to maximum concentration

${ }^{\mathrm{a}}$ Median 
ER versus placebo in the full analysis set [27, 28]. A step-down testing procedure, where sequential testing proceeded provided statistical significance $(p<0.05)$ was reached, was applied in both trials (for primary and secondary endpoints in the phase III study [28], and secondary endpoints only in the phase IIb study [27]); all subsequent data after the first non-significant finding were considered to be informative only [27, 28].

In the phase III study, the secondary endpoints were (in order of step-down testing) the area-under-effect (AUE) curve of the change in weekly mean ADP intensity scores from baseline to week $12\left(\mathrm{AUE}_{\text {week1-12 }}\right)$ for triamcinolone acetonide ER versus placebo, $\mathrm{AUE}_{\text {week } 1-12}$ for triamcinolone acetonide ER versus triamcinolone acetonide $\mathrm{CS}$, change in weekly mean ADP intensity scores from baseline to week 12 for triamcinolone acetonide ER versus triamcinolone acetonide CS, and $\mathrm{AUE}_{\text {week1-24 }}$ for triamcinolone acetonide ER versus placebo [28]. Other exploratory endpoints in the phase III study included changes from baseline in Western Ontario and McMaster Universities Osteoarthritis Index (WOMAC) scores to assess pain (WOMAC-A), stiffness (WOMAC-B) and physical function (WOMAC-C) and in Knee Injury and Osteoarthritis Outcome Score-assessed Quality of Life (KOOS-QOL) score and mean daily rescue medication use [28].

\subsubsection{Phase Illb Trial}

In the 24-week phase IIb study [27], the primary endpoint of change in ADP intensity at week 12 after a single IA injection of triamcinolone acetonide ER $32 \mathrm{mg}(n=104)$ in patients with OA pain of the knee was not met. However, significant improvements in ADP intensity versus placebo $(n=100)$ was seen at weeks $1-11(p \leq 0.036)$ and at week 13 $(p=0.039)$. Sensitivity analyses also indicated that triamcinolone acetonide ER treatment significantly $(p<0.05)$ improved pain relief compared with placebo during weeks $1-13$. Significant improvements $(p<0.05)$ in predefined secondary endpoints were also observed with triamcinolone acetonide ER versus placebo, including physical function as per WOMAC scoring and pain according to Patients' Global Impression of Change at weeks 4 and 8 [27].

\subsubsection{Phase III Trial}

The efficacy of triamcinolone acetonide ER $32 \mathrm{mg}(n=161)$ as a single $(5 \mathrm{~mL}) \mathrm{IA}$ injection versus placebo (saline) $(n=162)$ in patients with OA pain of the knee was evaluated in a 24-week, double-blind, phase III study [28]. As a secondary assessment, the study also evaluated the efficacy and safety of triamcinolone acetonide CS (1 mL IA injection) $40 \mathrm{mg}$ as an active comparator $(n=161)$. At baseline, the mean patient age was 62 (range $40-85$ years), and $\approx 50 \%$ of the patient population were obese [body mass index $\left.(\mathrm{BMI}) \geq 30 \mathrm{~kg} / \mathrm{m}^{2}\right]$ [28].
Significant improvements in pain relief were observed with triamcinolone acetonide ER compared with placebo in patients with pain from knee OA [28]. Triamcinolone acetonide ER recipients experienced significantly $(p<0.0001)$ greater LSM changes from baseline in ADP intensity score at week 12 compared with placebo [ -3.12 vs. -2.14 ; LSM between-group difference -0.98 (95\% CI -1.47 to -0.49 ); primary endpoint], suggesting a $\approx 50 \%$ improvement (Table 2). The between-group difference in changes in ADP intensity scores was significant $(p<0.05)$ between the triamcinolone acetonide ER and placebo groups every week over weeks 1-16 [28]. The results of prespecified sensitivity analyses, which allowed for missing-data imputation, were consistent with the findings of the primary analysis [27]. In the active comparator analysis, there was no significant betweengroup difference in changes from baseline to week 12 in ADP intensity scores between the triamcinolone acetonide ER and triamcinolone acetonide CS treatment groups (Table 2) [28].

In terms of ADP intensity scores, significantly more triamcinolone acetonide ER recipients than placebo recipients achieved improvements of $>30 \%$ (67.3 vs. 53.0\% at week $12 ; p<0.05$ at weeks $1-13$ ) and $>50 \%$ (52.3 vs. $37.1 \%$ at week $12 ; p<0.05$ at weeks $1-16$ and 18); no significant between-group difference was seen between triamcinolone acetonide ER and triamcinolone acetonide CS for these measures (data not reported) [28]. Similar onsets of action were seen between triamcinolone acetonide ER and triamcinolone acetonide CS recipients (median time to $>30 \%$ improvement in ADP intensity score of 4 and 3 days, respectively) [28].

The secondary endpoint of $\mathrm{AUE}_{\text {week1-12 }}$ also reflected an overall significant improvement over time with triamcinolone acetonide ER treatment relative to placebo (Table 2) [28]. However, $\mathrm{AUE}_{\text {week1-12 }}$ did not significantly differ between treatments with triamcinolone acetonide ER and triamcinolone acetonide CS. Although triamcinolone acetonide ER showed significant improvements in pain relief relative to placebo after 24 weeks following treatment with respect to $\mathrm{AUE}_{\text {week1-24 }}$ (Table 2), this outcome was deemed as informative only on the basis of the sequential testing used in the study [28].

Improvements were seen across the pre-specified exploratory endpoints of WOMAC and KOOS-QOL scores up to 12 weeks posttreatment with triamcinolone acetonide ER (Table 2) [28]. At week 12, treatment with triamcinolone acetonide ER had significantly improved pain (WOMAC-A), stiffness (WOMAC-B) and physical function (WOMAC-C) and KOOS-QOL scores compared with placebo (all $p<0.0001$ ) and with triamcinolone acetonide CS (all $p<0.05)$ [28].

The number of daily rescue medication tablets used per week was significantly lower in the triamcinolone acetonide ER group than the triamcinolone acetonide CS group overall [between-group LSM difference -0.50 (95\% CI -0.78 to $-0.21) ; p<0.001]$ and at weeks $2-16,19$ and $20(p \leq 0.027)$ [28]. 
Table 2 Efficacy of triamcinolone acetonide ER $32 \mathrm{mg}$ as a single IA injection in patients with pain from osteoarthritis of the knee in a randomized, double-blind, phase III trial; data are least square means [28]

\begin{tabular}{|c|c|c|c|c|c|}
\hline Endpoint & $\begin{array}{l}\text { TA-ER } \\
(n=161)\end{array}$ & $\begin{array}{l}\text { PL } \\
(n=162)\end{array}$ & $\begin{array}{l}\text { BGD TA-ER vs. PL } \\
(95 \% \mathrm{CI})\end{array}$ & $\begin{array}{l}\text { TA-CS } \\
(n=161)\end{array}$ & $\begin{array}{l}\text { BGD TA-ER vs. TA-CS } \\
(95 \% \mathrm{CI})\end{array}$ \\
\hline
\end{tabular}

\begin{tabular}{llllll}
\hline Key endpoints $^{\mathbf{a}}$ & & & & \\
\hline ADP change from BL at week 12 & -3.12 & -2.14 & $-0.98(-1.47,-0.49)^{* * *}$ & -2.86 & $-0.26(-0.74,0.23)$ \\
\hline AUE $_{\text {week1-12 }}$ & -247.3 & -145.3 & $-102.0(-136.8,-67.3)^{* * *}$ & -231.9 & $-15.3(-49.8,19.2)$ \\
\hline AUE $_{\text {week1-24 }}$ & -432.5 & -297.0 & $-135.5(-205.9,-65.2)^{* * \mathrm{~b}}$ & $\mathrm{NA}$ & $\mathrm{NA}$ \\
\hline Exploratory endpoints $^{\mathbf{c}}$ & & & & & \\
\hline WOMAC-A (pain) & $\mathrm{NA}$ & $\mathrm{NA}$ & $-0.37(-0.55,-0.20)^{* * *}$ & $\mathrm{NA}$ & $-0.17(-0.34,-0.00)^{*}$ \\
\hline WOMAC-B (stiffness) & & & $-0.44(-0.63,-0.25)^{* * *}$ & $-0.23(-0.42,-0.04)^{*}$ \\
\hline WOMAC-C (physical function) & & & $-0.38(-0.54,-0.21)^{* * *}$ & $-0.22(-0.38,-0.05)^{*}$ \\
\hline KOOS-QOL & & $+8.97(+4.37,+13.57)^{* * *}$ & $+5.42(+0.78,+10.06)^{*}$ \\
\hline
\end{tabular}

$A D P$ average daily pain, $A U E$ area under effect, $B G D$ between group difference, $B L$ baseline, $C I$ confidence interval, $C S$ crystalline suspension, $E R$ extended-release, $I A$ intra-articular, KOOS-QOL Knee Injury and Osteoarthritis Outcome Score-Quality of Life, $L S M$ least squares mean, $N A$ not available, $P L$ placebo, TA triamcinolone acetonide, WOMAC Western Ontario and McMaster Universities Osteoarthritis Index

$* p<0.05, * * p<0.001, * * * p<0.0001$ vs. PL

a Primary endpoint: LSM change from BL to week 12 in ADP with TA-ER vs. PL in the full analysis set. Secondary endpoints (in step-down

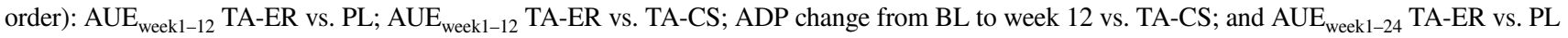
${ }^{\mathrm{b}} p$-value considered informative only as the previous comparison in step-down testing (AUE $\mathrm{week} 1-12_{2}$ for TA-ER vs. TA-CS) was not significant

${ }^{\mathrm{c}}$ Data recorded at week 12

\subsection{Repeat Administration}

The efficacy of repeat administration of IA triamcinolone acetonide ER was investigated in a 52-week, phase IIIb, single-arm, open-label safety study in patients aged $\geq 40$ years with knee OA $(n=208)$ [29]. Two IA injections of triamcinolone acetonide ER $32 \mathrm{mg}$ were administered, with the second dose administered using a flexible dosing schedule with the timing based on patient response [29].

Eligible patients had symptomatic knee OA for $\geq 6$ months, an ACR clinical and radiologic diagnosis of OA, a KellgrenLawrence grade of 2-4, a WOMAC-A total sum score of $\geq 6$, and index-knee pain for $\geq 15$ days during the month prior to the study [29]. Patients were eligible to receive a second injection of triamcinolone acetonide ER $32 \mathrm{mg}$ if they had initially experienced clinical benefits from the first injection, were clinically indicated to receive a second dose, and had no major safety concerns after receiving the first dose (administered on day 1 of the study); the flexible dosing schedule allowed for patients to receive the second injection at weeks 12, 16, 20 or 24 if these requirements were met. Those who did not meet these response requirements terminated the study early. Most patients (86.1\%) received a second injection of triamcinolone acetonide ER; the median time to second injection was 16.6 weeks [29].

Patients who received a second injection $(n=179)$ of triamcinolone acetonide ER experienced generally similar improvements in pain, stiffness and function (as per LSM changes of WOMAC scores) and KOOS-QOL to those seen with the first injection [29]. At 4 weeks post-injection, the improvements in pain $(-1.11$ to -1.38 vs. -1.38 to -1.56 after second and first injections), stiffness $(-1.37$ to -1.87 vs. -1.65 to -1.85$)$ and function $(-1.23$ to -1.45 vs. -1.47 to -1.54 ) were similar. Furthermore, the duration of clinical benefit observed following the second injection was generally similar to that following the first injection (up to 24 weeks after each injection) across the WOMAC subscales for pain (WOMAC-A: -0.07 to -0.21 vs. -0.42 to -0.63 for first and second injections), stiffness (WOMAC-B: -0.15 to -0.44 vs. -0.57 to -0.83 ) and function (WOMAC-C: 0.00 to -0.42 vs. -0.35 to -0.74 ). At 4 weeks after the first and second injections, most patients were considered moderate ( $\geq 30 \%$ improvement) or substantial $(\geq 50 \%$ improvement) WOMAC-A, -B, -C and KOOS-QOL responders [29].

\section{Tolerability}

\subsection{Single Administration}

A single IA injection of triamcinolone acetonide ER was generally well tolerated in patients with OA pain of the knee across the single administration clinical trials [26-28]. The tolerability profile of triamcinolone acetonide ER did not noticeably differ from that of placebo $[27,28]$ or triamcinolone acetonide CS [28], and most adverse events (AEs) in all treatment groups were of mild or moderate severity. No patients in the triamcinolone acetonide ER treatment groups withdrew from the studies due to a treatment-related AE (TRAE), and no deaths occurred [27, 28].

In the phase III study, similar proportions of patients experienced at least one $\mathrm{AE}$ in each of the triamcinolone acetonide ER, placebo and triamcinolone acetonide CS 
groups (55.3, 53.1 and 56.5\%, respectively), with most AEs being of grade $1(23.0,20.4$ and $24.8 \%)$ or grade 2 (28.0, 29.6 and 29.2\%) severity [28]. Almost half of all AEs were deemed to be unrelated to the study treatment (42.9 for triamcinolone acetonide ER vs. 47.5 and $48.4 \%$ for placebo and triamcinolone acetonide CS), with a low proportion (6.2 vs. 1.9 and $2.5 \%$, respectively) considered possibly, probably or definitely treatment-related [28]. The most common AEs ( $>5 \%$ in any group) with triamcinolone acetonide ER were arthralgia in any joint (14.3 vs. 12.3 and $7.5 \%$ for placebo and triamcinolone acetonide CS, respectively), headache ( 8.7 vs. 8.0 and $9.3 \%$ ) and back pain (5.6 vs. 5.6 and $7.5 \%)$. The frequency of serious AEs was low with triamcinolone acetonide ER (3.1 vs. 1.9 and $2.5 \%$ for placebo and triamcinolone acetonide CS, respectively) and none were deemed to be treatment-related in any treatment group [28].

Index knee-related AEs occurred in 18.6, 12.3 and 9.9\% of patients treated with triamcinolone acetonide ER, placebo and triamcinolone acetonide CS, respectively, with the majority of these unrelated to treatment [28]. Only 3.1, 1.9 and $0.6 \%$ were determined to be possibly, probably or definitely treatment-related, although none were consistent with postinjection OA flare. Worsening of joint-space narrowing was uncommon across the treatment groups (incidence of 5.0, 4.1 and 3.5\% with triamcinolone acetonide ER, placebo and triamcinolone acetonide CS, respectively), with worsening only increased by 1 grade in these patients, except for one grade $0 \rightarrow 2$ worsening in a placebo recipient. There was no radiographic evidence of rapidly progressive $\mathrm{OA}$ in patients treated with triamcinolone acetonide ER [28].

Joint infections were not reported in any patient; the incidence of non-joint infections was 16.8, 19.1 and 21.7\% (in patients treated with triamcinolone acetonide ER, placebo and triamcinolone acetonide CS, respectively), including upper respiratory tract infection, nasopharyngitis, influenza, sinusitis, and viral upper respiratory tract infections [28].

Data from a pooled analysis $[11,30]$ of the phase II and III trials [26-28] in patients receiving IA triamcinolone acetonide ER $32 \mathrm{mg}(n=424)$ support the findings of the individual trials. The most common $(\geq 1 \%)$ treatmentemergent adverse reactions (ARs) in patients treated with triamcinolone acetonide ER $32 \mathrm{mg}(n=424)$ compared with placebo $(n=262)$ were sinusitis, cough and contusions $(2$ vs. $1 \%$ for each $\mathrm{AR}$ ); the most common treatment-emergent index knee-related ARs were joint swelling ( 3 vs. $2 \%$ ) and contusions (2 vs. 1\%) [11]. Although patients with a BMI of $>40 \mathrm{~kg} / \mathrm{m}^{2}$ were excluded from the analysis, there was no positive correlation between BMI (median and mean BMI both $>30 \mathrm{~kg} / \mathrm{m}^{2}$ ) and toxicity with triamcinolone acetonide ER treatment, suggesting that its use should not be limited in patients with very high BMI [30].

\subsection{Repeat Administration}

The tolerability profile of IA triamcinolone acetonide ER after up to two injections was generally consistent with that seen in the single administration phase IIb and III clinical studies [29]. Most AEs (total AE incidence rate 41.9 and 35.2\% after the first and second injections) were considered to be unrelated to treatment, non-serious, and/or grade 1 or 2 . The most frequent $\mathrm{AE}$ in patients who received two injections were arthralgia in any joint (10.6 and $19.0 \%$ after the first and second injections). After the first injection, two cases of arthralgia in the index knee were considered treatment-related (at 2 and 22 days after injection). Although three patients experienced arthralgia after the second injection (at 58, 80 and 146 days after injection) and discontinued treatment, none of these cases were considered treatment-related [29].

No unexpected treatment emergent AEs were reported and, in those who had baseline and end-of-study radiographs (92.2\% of patients), no cases of chondrolysis, osteonecrosis, insufficiency fractures or clinically significant subchondral bone changes were recorded at the end of study (week 52 or at discontinuation) [29].

\section{Dosage and Administration}

Triamcinolone acetonide ER is approved in the USA for the management of OA pain of the knee, and is administered as a single ( $5 \mathrm{~mL}$ ) IA injection to deliver $32 \mathrm{mg}$ of triamcinolone acetonide (determined to be the delivered dose from $40 \mathrm{mg}$ ) [11]. Triamcinolone acetonide ER is supplied as a single-dose kit containing a vial of the triamcinolone acetonide ER as a microsphere powder that must be diluted with the supplied diluent; injections should be made promptly after preparation to avoid settling of the suspension [11].

Triamcinolone acetonide ER is for IA use only and should not be administered via other routes as these are yet to be evaluated [11]. The efficacy and safety of triamcinolone acetonide ER for management of OA pain of shoulder and hip have not been evaluated. Triamcinolone acetonide ER is not suitable for use in small joints, such as the hand. IA corticosteroids should not be injected into an infected site, and IA corticosteroid injections into unstable joints is generally not recommended [11].

Local prescribing information should be consulted for detailed information regarding contraindications, use in special patient populations, drug interactions, warnings and precautions.

\section{Current Status}

Current treatment guidelines for the management of OA pain of the knee recommend the combined use of non-pharmacological interventions (such as exercises and manual therapy [31]) with pharmacological interventions [32, 33]. The ACR 
and AAOS guidelines recommend oral and topical nonsteroidal anti-inflammatory drugs (NSAIDs) and tramadol $[32,33]$; in addition, the ACR guidelines recommend the use of paracetamol and IA corticosteroids [33]. Although IA corticosteroids are an important treatment option, their efficacy is typically short-lived (Sect. 1), with evidence for their longer-term effectiveness inconclusive [7, 9, 32]. Other treatment types are currently being further investigated for knee $\mathrm{OA}$, such as neuroablation procedures (e.g. cryoneurolysis [34]) and platelet-rich plasma (PRP) therapy; PRP therapy is largely used in a hospital setting and is being assessed in its effectiveness and feasibility in primary care [35, 36].

In contrast with triamcinolone acetonide CS, the formulation of triamcinolone acetonide ER allows a slower release of triamcinolone acetonide in the injection site, not only prolonging its presence in the synovium but also decreasing its systemic absorption (Sect. 3). Corticosteroid use, including IA administration, in patients with T2DM may result in a transient increase in blood glucose levels and therefore dosage adjustments of antidiabetic agents may be required when undergoing corticosteroid-based therapy [11]. Patients with T2DM receiving triamcinolone acetonide ER experienced a significantly smaller blood glucose elevation compared with those receiving triamcinolone acetonide CS and a negligible impact on their glycaemic control (Sect. 2).

Treatment with a single IA injection of triamcinolone acetonide ER $32 \mathrm{mg}$ provided significant pain relief in patients with OA pain of the knee relative to placebo with respect to ADP scoring in clinical trials (Sect. 4), and provided a similar level of pain relief to that of a single injection of IA triamcinolone acetonide CS (Sect. 4.1.2). When assessed using the OA-specific WOMAC instrument, triamcinolone acetonide ER recipients experienced significant improvements in pain, stiffness and physical function compared with triamcinolone acetonide CS and placebo, as well as in KOOS-QOL in the phase III trial (Sect. 4.1.2). The differences seen between ADP and WOMAC pain findings with IA triamcinolone acetonide ER may be due to a greater degree of responsiveness with WOMAC scoring, where pain subscale scores are based on a multi-item tool measuring disease-specific outcomes, rather than the single-item pain intensity measure of ADP [37].

IA triamcinolone acetonide ER had a similar tolerability profile to those seen with IA triamcinolone acetonide CS and placebo, and was generally well tolerated with up to 24 weeks of treatment, with most AEs being of mild or moderate severity (Sect. 5.1). The majority of index knee-related AEs with triamcinolone acetonide ER were considered unrelated to treatment, worsening of joint-space narrowing was uncommon and no radiographic evidence of rapidly progressive OA was observed over the 24-week study period (Sect. 5.1). Longer-term data would be beneficial in further evaluating the tolerability of triamcinolone acetonide ER in treating OA pain of the knee. Head-to-head trials of triamcinolone acetonide ER and triamcinolone acetonide
CS would also be helpful in establishing the relative efficacy and tolerability of these formulations. Given that triamcinolone acetonide CS is approved for use in multiple joints [38], future investigations of triamcinolone acetonide ER in osteoarthritic joints other than the knee may be valuable.

Findings from a single-arm phase IIIlb study (Sects. Sects. 4.2 and 5.2) suggest that a repeat administration of triamcinolone acetonide ER may be similarly efficacious to an initial injection without further increasing the risk for AEs, with no radiographic evidence of deleterious effects on cartilage or other aspects of joint structure with the repeat administration. Further investigation into the tolerability and efficacy of repeat administration of triamcinolone acetonide ER would be of interest, namely with longer-term and/or placebo-controlled studies.

In conclusion, triamcinolone acetonide ER was effective and well tolerated in phase IIb and III studies, and its approval expands the treatment options available for the management of OA pain of the knee.

Data Selection Triamcinolone acetonide: 123 records identified

Duplicates removed

Excluded during initial screening (e.g. press releases; news reports; not relevant drug/indication; preclinical study; reviews; case reports; not randomized trial)

Excluded during writing (e.g. reviews; duplicate data; small patient number; nonrandomized/phase I/II trials)

Cited efficacy/tolerability articles Cited articles not efficacy/tolerability 6

Search Strategy: EMBASE, MEDLINE and PubMed from 1946 to present. Clinical trial registries/databases and websites were also searched for relevant data. Key words were Triamcinolone acetonide, osteoarthritis, intra-articular, knee. Records were limited to those in English language. Searches last updated 22 February 2019

Acknowledgements During the peer review process, the manufacturer of triamcinolone acetonide ER was also offered an opportunity to review this article. Changes resulting from comments received were made on the basis of scientific and editorial merit.

\section{Compliance with Ethical Standards}

Funding The preparation of this review was not supported by any external funding.

Conflicts of interest Julia Paik, Sean Duggan and Susan Keam are salaried employees of Adis/Springer and declare no relevant conflicts of interest.

Open Access This article is distributed under the terms of the Creative Commons Attribution-NonCommercial 4.0 International License (http://creativecommons.org/licenses/by-nc/4.0/), which permits any noncommercial use, duplication, adaptation, distribution and reproduction in any medium or format, as long as you give appropriate credit to the original author(s) and the source, provide a link to the Creative Commons license and indicate if changes were made. 


\section{References}

1. Vos T, Flaxman AD, Naghavi M, et al. Years lived with disability (YLDs) for 1160 sequelae of 289 diseases and injuries 19902010: a systematic analysis for the Global Burden of Disease Study 2010. Lancet. 2012;380(9859):2163-96.

2. Osteoarthritis Research Society International. Osteoarthritis: a serious disease, a white paper submitted to the U.S. Food and Drug Administration. 2016. https://www.oarsi.org/. Accessed 22 Feb 2019.

3. Hawker GA, Stewart L, French MR, et al. Understanding the pain experience in hip and knee osteoarthritis: an OARSI/OMERACT initiative. Osteoarthr Cartil. 2008;16(4):415-22.

4. Nuesch E, Dieppe P, Reichenbach S, et al. All cause and disease specific mortality in patients with knee or hip osteoarthritis: population based cohort study. BMJ. 2011;342:d1165.

5. Sellam J, Berenbaum F. The role of synovitis in pathophysiology and clinical symptoms of osteoarthritis. Nat Rev Rheumatol. 2010;6(11):625-35.

6. Hochberg MC, Altman RD, Brandt KD, et al. Guidelines for the medical management of osteoarthritis. Part II. Osteoarthritis of the knee. American College of Rheumatology. Arthritis Rheum. 1995;38(11):1541-6.

7. Juni P, Hari R, Rutjes AW, et al. Intra-articular corticosteroid for knee osteoarthritis. Cochrane Database Syst Rev. 2015(10):CD005328

8. Koenig KM, Ong KL, Lau EC, et al. The use of hyaluronic acid and corticosteroid injections among Medicare patients with knee osteoarthritis. J Arthroplast. 2016;31(2):351-5.

9. da Costa BR, Hari R, Juni P. Intra-articular corticosteroids for osteoarthritis of the knee. JAMA. 2016;316(24):2671-2.

10. Habib GS. Systemic effects of intra-articular corticosteroids. Clin Rheumatol. 2009;28(7):749-56.

11. Flexion Therapeutics Inc. ZILRETTA (triamcinolone acetonide extended-release injectable suspension): US prescribing information. 2017. https://www.accessdata.fda.gov/. Accessed 22 Feb 2019.

12. US Center for Drug Evaluation and Research. Non-clinical review(s) (Zilretta). 2017. https://www.accessdata.fda.gov/. Accessed 22 Feb 2019.

13. Anderson JM, Shive MS. Biodegradation and biocompatibility of PLA and PLGA microspheres. Adv Drug Deliv Rev. 1997;28(1):5-24.

14. Flexion Therapeutics Inc. ZILRETTA ${ }^{\circledR}$ (triamcinolone acetonide extended-release injectable suspension). 2019. https://flexionthe rapeutics.com/our-product/. Accessed 22 Feb 2019.

15. Spenlehauer G, Vert M, Benoit JP, et al. In vitro and in vivo degradation of poly(D, L lactide/glycolide) type microspheres made by solvent evaporation method. Biomaterials. 1989;10(8):557-63.

16. Jackson D, Cotton L, Turkington M, et al. Physical and chemical compatibility of extended-release triamcinolone acetonide (TAER) with common local anesthetics. Adv Ther. 2019. https://doi. org/10.1007/s12325-019-0878-2.

17. Scherer J, Rainsford KD, Kean CA, et al. Pharmacology of intraarticular triamcinolone. Inflammopharmacology. 2014;22(4):201-17.

18. US Center for Drug Evaluation and Research. Clinical pharmacology and biopharmaceutics review (Zilretta). 2017. https://www. accessdata.fda.gov/. Accessed 22 Feb 2019.

19. Wernecke C, Braun HJ, Dragoo JL. The effect of intra-articular corticosteroids on articular cartilage: a systematic review. Orthop J Sports Med. 2015;3(5):2325967115581163.

20. Kumar A, Bendele AM, Blanks RC, et al. Sustained efficacy of a single intra-articular dose of FX006 in a rat model of repeated localized knee arthritis. Osteoarthr Cartil. 2015;23(1):151-60.

21. Williamson TL, Walz A, Garlick D, et al. Systemic and local effects following intra-articular injection of FX006, an extended release, PLGA microsphere formulation of triamcinolone acetonide: results from two nonclinical toxicity studies in dogs (abstract no. 713). Osteoarthr Cartil. 2017;25(Suppl. 1):S431-S2.

22. Bodick N, Williamson T, Strand V, et al. Local effects following single and repeat intra-articular injections of triamcinolone acetonide extended-release: results from 3 nonclinical toxicity studies in dogs. Rheumatol Ther. 2018;5(2):475-98.

23. Russell SJ, Sala R, Conaghan PG, et al. Triamcinolone acetonide extended-release in patients with osteoarthritis and type 2 diabetes: a randomized, phase 2 study. Rheumatology (Oxford). 2018;57(12):2235-41.

24. Kraus VB, Conaghan PG, Aazami HA, et al. Synovial and systemic pharmacokinetics (PK) of triamcinolone acetonide (TA) following intra-articular (IA) injection of an extended-release microsphere-based formulation (FX006) or standard crystalline suspension in patients with knee osteoarthritis (OA). Osteoarthr Cartil. 2018;26(1):34-42.

25. Collegium Pharmaceutical Inc. AllerNaze (triamcinolone acetonide nasal spray): US prescribing information. 2009. https://www. accessdata.fda.gov/. Accessed 22 Feb 2019.

26. Bodick N, Lufkin J, Willwerth C, et al. An intra-articular, extendedrelease formulation of triamcinolone acetonide prolongs and amplifies analgesic effect in patients with osteoarthritis of the knee: a randomized clinical trial. J Bone Jt Surg Am. 2015;97(11):877-88.

27. Conaghan PG, Cohen SB, Berenbaum F, et al. Brief report: a phase IIb trial of a novel extended-release microsphere formulation of triamcinolone acetonide for intraarticular injection in knee osteoarthritis. Arthritis Rheumatol. 2018;70(2):204-11.

28. Conaghan PG, Hunter DJ, Cohen SB, et al. Effects of a single intra-articular injection of a microsphere formulation of triamcinolone acetonide on knee osteoarthritis pain: a double-blinded, randomized, placebo-controlled, multinational study. J Bone Jt Surg Am. 2018;100(8):666-77.

29. Spitzer AI, Richmond JC, Kraus VB, et al. Safety and efficacy of repeat administration of triamcinolone acetonide extended-release in osteoarthritis of the knee: a phase $3 \mathrm{~b}$, open-label study. Rheumatol Ther. 2019. https://doi.org/10.1007/s40744-019-0140-z.

30. US Center for Drug Evaluation and Research. Summary review (Zilretta). 2018. https://www.accessdata.fda.gov/. Accessed 22 Feb 2019.

31. Henriksen M, Klokker L, Graven-Nielsen T, et al. Association of exercise therapy and reduction of pain sensitivity in patients with knee osteoarthritis: a randomized controlled trial. Arthritis Care Res (Hoboken). 2014;66(12):1836-43.

32. American Academy of Orthopaedic Surgeons. Treatment of osteoarthritis of the knee: evidence-based guideline, 2nd edn. 2013. https://www.aaos.org/. Accessed 22 Feb 2019.

33. Hochberg MC, Altman RD, April KT, et al. American College of Rheumatology 2012 recommendations for the use of nonpharmacologic and pharmacologic therapies in osteoarthritis of the hand, hip, and knee. Arthritis Care Res (Hoboken). 2012;64(4):465-74.

34. Radnovich R, Scott D, Patel AT, et al. Cryoneurolysis to treat the pain and symptoms of knee osteoarthritis: a multicenter, randomized, double-blind, sham-controlled trial. Osteoarthr Cartil. 2017;25(8):1247-56.

35. Dhillon MS, Patel S, John R. PRP in OA knee-update, current confusions and future options. SICOT-J. 2017;3:27.

36. Glynn LG, Mustafa A, Casey M, et al. Platelet-rich plasma (PRP) therapy for knee arthritis: a feasibility study in primary care. Pilot Feasibility Study. 2018;4:93.

37. Dworkin RH, Peirce-Sandner S, Turk DC, et al. Outcome measures in placebo-controlled trials of osteoarthritis: responsiveness to treatment effects in the REPORT database. Osteoarthr Cartil. 2011;19(5):483-92.

38. Bristol-Myers Squibb. KENALOG-40 injection (triamcinolone acetonide injectable suspension): US prescribing information. 2018. https://www.bms.com/. Accessed 22 Feb 2019. 